\title{
Epidemiology of organising pneumonia in Iceland
}

G Gudmundsson, O Sveinsson, H J Isaksson, S Jonsson, H Frodadottir, T Aspelund

See end of article for authors' affiliations

$\ldots . .$.

Correspondence to: Dr G Gudmundsson, Department of Respiratory

Medicine, Allergy and

Sleep, Landspitali

University Hospital, E-7

Fossvogur, IS-108

Reykjavik, Iceland;

ggudmund@landspitali.is

Received 21 January 2006

Accepted 7 June 2006

Published Online First

29 June 2006
Background: Cryptogenic organising pneumonia (COP) has also been called idiopathic bronchiolitis obliterans organising pneumonia. In secondary organising pneumonia (SOP) the causes can be identified or it occurs in a characteristic clinical context. The aim of this study was to determine the incidence and epidemiological features of COP and SOP nationwide in lceland over an extended period.

Methods: A retrospective study of organising pneumonia (OP) in lceland over 20 years was conducted and the epidemiology and survival were studied. All pathological reports of patients diagnosed with or suspected of having COP or SOP in the period 1984-2003 were identified and the pathology samples were re-evaluated using strict diagnostic criteria.

Results: After re-evaluation, 104 patients fulfilled the diagnostic criteria for OP (58 COP and 46 SOP). The mean annual incidence of OP was 1.97/100 000 population (1.10/100 000 for COP and 0.87/ 100000 for SOP). The mean age at diagnosis was 67 years with a wide age range. The most common causes of death were lung diseases other than OP, and only one patient died from OP. Patients with OP had a lower rate of survival than the general population, but there was no statistical difference between COP and SOP.

Conclusions: The incidence of $O P$ is higher than previously reported, suggesting that $O P$ needs to be considered as a diagnosis more often than has been done in the past.
T he diagnosis of organising pneumonia (OP) is based on a characteristic histological pattern in the presence of certain clinical and radiological features. The histological pattern is characterised by polypoid connective tissue masses composed of myxoid fibroblastic tissue resembling granulation tissue filling the lumens of terminal and respiratory bronchioles and extending in a continuous fashion into the alveolar ducts and alveoli, representing an OP. ${ }^{1}$ It is called cryptogenic organising pneumonia (COP) when no definite cause or characteristic clinical context is found. ${ }^{2}$ In secondary OP (SOP) causes such as infection can be identified or it occurs in a characteristic clinical context such as connective tissue disorder. A classification of idiopathic interstitial pneumonias has recently been published. ${ }^{2}$ COP is one of several subtypes and represents what has also been called idiopathic bronchiolitis obliterans organising pneumonia (BOOP). Most reports of BOOP/COP are small case series or single case reports. ${ }^{3}{ }^{4}$ The epidemiology is therefore not well described and not much is known about the incidence of these diseases.

A study was undertaken to compare the epidemiology and outcome of cases of COP and SOP over a 20 year period (1984-2003) in a nationwide study in Iceland. The incidence rate, survival, and causes of death from COP and SOP during this period were determined. This is the first nationwide survey to examine the incidence of both COP and SOP in the same time period, using strict diagnostic criteria in a homogenous population. This is a retrospective study with no referral bias affecting the results.

\section{METHODS}

\section{Clinical dato}

Information on all patients diagnosed with OP from 1984 to 2003 was obtained by specific ICD codes from computerised hospital records and from specific codes from computerised pathology reports. The International Classification of Diseases (ICD) is designed for the classification of morbidity and mortality information for statistical purposes, and for indexing hospital records by disease and operations for data storage and retrieval. ICD is published by the World Health Organization (WHO). The records of patients with the following codes were evaluated: J68, J70, J84 and J98. All lung specialists in Iceland $(n=14$ at the end of the study period) were also asked to provide information about patients diagnosed with OP during this time period. All gave information on patients with OP. Clinical data were collected from clinical records from hospitals and private offices using a standardised data sheet and were reviewed by two of the investigators.

The population of Iceland increased during the study period from 239498 in 1984 to 289272 in 2003, an increase of $17.2 \%$. About $60 \%$ of the population lives in the capital, Reykjavik, and the greater Reykjavik area. All information regarding the size of the population, age, sex distribution, and cause of death (death certificates) was obtained from Statistics Iceland (the National Statistical Institute of Iceland).

The study was approved by the National Data Protection Agency of Iceland and National Ethics Committee, Reykjavik.

\section{Pathology}

All pathology specimens in Iceland are evaluated at the Department of Pathology at Landspitali University Hospital in Reykjavik and the Department of Pathology at Akureyri Regional Hospital. Both departments use the SNOMED (Systematised Nomenclature of Medicine) coding system. The following codes were used: T26000-T28900, M45700, which is a morphological code for OP and M40000-M49990, D7325-7705 to cover all other inflammatory and interstitial lung disorders. SNOMED is published by the College of American Pathologists.

All specimens were reviewed by one pathologist (HJI) and those classified as OP were used in the study. Glass slides and paraffin tissue blocks are stored indefinitely. When needed,

Abbreviations: COP, cryptogenic organising pneumonia; ILD, interstitial lung disease; OP, organising pneumonia; SOP, secondary organising pneumonia 
Table 1 Number of patients, sex, median age, and mean annual incidence of organising pneumonia (OP)

\begin{tabular}{|c|c|c|c|}
\hline & Cryptogenic OP & Secondary OP & Total \\
\hline No of patients & 58 & 46 & 104 \\
\hline$M / F$ & $31 / 27$ & $25 / 21$ & $56 / 48$ \\
\hline $\begin{array}{l}\text { Median (range) age at diagnosis } \\
\text { (years) }\end{array}$ & $66(15-87)$ & $70(26-83)$ & $67(15-87)$ \\
\hline \multicolumn{4}{|l|}{$\begin{array}{l}\text { Mean }(95 \% \mathrm{Cl}) \text { annual incidence } \\
\text { per } 100000\end{array}$} \\
\hline Men & $1.13(0.79$ to 1.62$)$ & $0.94(0.64$ to 1.40$)$ & $2.08(1.60$ to 2.71$)$ \\
\hline Women & $1.06(0.73$ to 1.53$)$ & $0.80(0.52$ to 1.22$)$ & $1.86(1.41$ to 2.46$)$ \\
\hline Total & $1.10(0.85$ to 1.42$)$ & $0.87(0.65$ to 1.16$)$ & 1.97 (1.62 to 2.39$)$ \\
\hline
\end{tabular}

re-cuts and special stains were made. After review, some cases were excluded from the study. To make a diagnosis of $\mathrm{OP}$, the pattern of OP had to be prominent and not an accessory finding of another well defined pattern of idiopathic interstitial pneumonia. The diagnosis of OP was ruled out if biopsies showed prominent cellular and significant interstitial fibrosis, fibroblastic foci, granulomas, hyaline membranes, vasculitis, and prominent eosinophilia. ${ }^{2}$

\section{Inclusion criteria}

All patients enrolled in the study were required to meet the following criteria: (1) radiological lung infiltrates or nodules; (2) lung biopsy specimen (transbronchial or surgical) confirming OP; (3) a well documented improvement that was either spontaneous or following immunosuppressive treatment (usually corticosteroids). Clinical criteria were applied to patients after screening of records. The pathological reports were then evaluated. Some cases did not qualify for pathology and were therefore excluded. Clinical criteria or the clinical context of the case were used to classify cases as SOP; if none of these criteria were met, the case was classified as COP:

- Post infectious: if the patient had symptoms and signs consistent with the diagnosis of a respiratory infection and positive cultures or serology or antigen detection for a known bacteria, virus or other pathogen at the time of diagnosis.

- Drug related: if the onset of OP was in relation to drug treatment and the drug was previously known to cause OP and no other cause could be found.

- Radiotherapy related: if the onset of OP was in relation to radiotherapy to the chest or surrounding area and no other cause could be found.

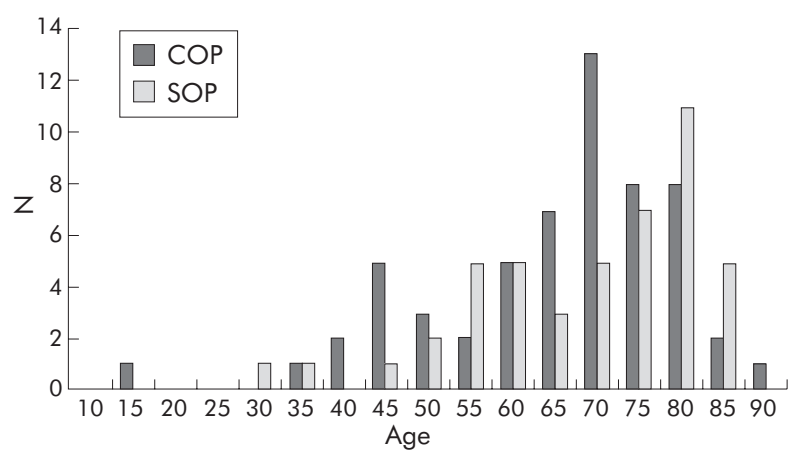

Figure 1 Age distribution for organising pneumonia in Iceland. COP, cryptogenic organising pneumonia; SOP, secondary organising pneumonia.
- Rheumatological disorder: if the patient had a previous diagnosis of rheumatological disease and no other cause could be found.

\section{Causes of death}

Three methods were used to determine the cause of death: (1) necropsy reports; (2) hospital records; and (3) death certificates from Statistics Iceland. The time to death was taken from the time of diagnosis.

\section{Statistical analysis}

The calculation of the annual incidence per 100000 was based on the number of inhabitants on 1 July of each year. It was assumed that the number of cases each year follows a Poisson distribution, and the incidence rates were analysed with a Poisson regression model using age, sex, and period specific population size as an offset term. The mean annual increase in incidence was estimated by a linear Poisson regression model both overall and by age group. The Poisson model was used to test for interaction between age group and increase in incidence. Age at diagnosis was compared between time periods by ANOVA. Mortality between patients and the population was compared using the standardised mortality ratio approach. ${ }^{5}$ It is based on the total observed deaths versus the total expected deaths estimated by computing individual expected cumulative hazards. Survival rates for the study group were estimated using the KaplanMeier method. Survival rates for a reference population were based on the cohort expected survival approach of Hakulinen as outlined in Therneau and Grambsch. ${ }^{6}$ Both the individual expected cumulative hazards and the expected cohort survival rates were constructed from age and sex matched survival rates in a national life table from Statistics Iceland, 1993. A p value of $<0.05$ was considered significant. Data analysis was performed using SAS software version 9.1 (SAS Institute Inc, Cary, NC, USA).

\section{RESULTS}

Using the strict criteria, 104 patients with OP were identified during the study period. Their demographic data are shown in table 1. Fifty eight patients had COP and 46 had SOP, with similar numbers of men and women and median age at

\begin{tabular}{lll} 
Table 2 & Mean (SD) age by study period \\
\hline Time period & $\begin{array}{l}\text { Number } \\
\text { diagnosed }\end{array}$ & Mean (SD) age \\
\hline $1984-1989$ & 12 & $64.8(16.4)$ \\
$1990-1994$ & 16 & $63.4(13.4)$ \\
$1995-1999$ & 41 & $63.1(14.0)$ \\
$2000-2003$ & 35 & $67.1(14.2)$ \\
\hline
\end{tabular}




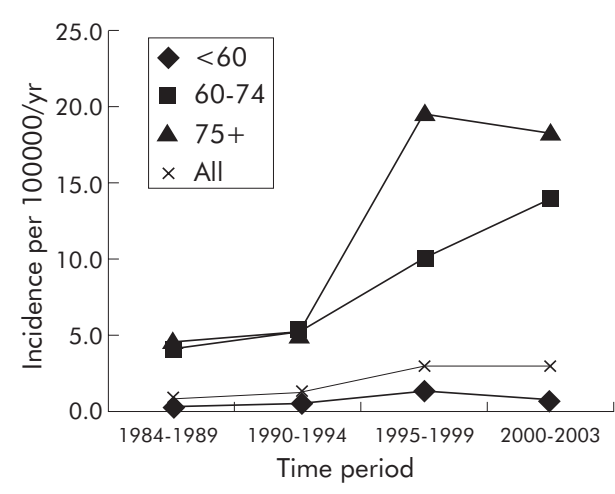

Figure 2 Incidence of organising pneumonia by year and age.

diagnosis in the two groups. Overall, the mean (SD) age was 67 (14) years. Figure 1 shows the wide age distribution found in the study (range 15-87) years. A comparison of the age distribution between the time periods 1984-8, 1989-94, 1995-9, and 2000-3 showed a non-significant difference $(\mathrm{p}=0.64$, ANOVA). During the study period the mean age at diagnosis remained similar but was found to be higher from year 2000 (table 2).

The incidence of both COP and SOP increased during the study period, but COP increased more consistently than SOP. The incidence of OP increased significantly $(p<0.0001)$. The annual incidence of OP is summarised in fig 2 by time period and age group. The mean annual incidence of OP for 19842003 was 1.97 (95\% CI 1.63 to 2.39) for the period 1984-2003, but for the period $2000-3$ it was 3.06 (95\% CI 2.20 to 4.26 ). On average, the incidence of OP rose by $8.1 \%$ (95\% CI 4.1 to 12.2) per year (table 3 ). The increase in the incidence of OP did not differ between the age groups $(p=0.99)$.

Forty five patients died during the study and follow up period. The most common causes of death were lung diseases other than OP; of these, chronic obstructive pulmonary disease was most common. Other causes of death are shown in table 4.

Only one patient died from OP; this patient had the rapidly progressive form. One patient died from steroid related complications. The mortality rates in COP and SOP were significantly higher than in the general population (fig 3). The standardised mortality ratio for OP was 2.7 (SE 0.4); for COP the standardised mortality ratio was 2.6 (SE 0.6) and for SOP 2.9 (SE 0.6). There was no difference in mortality between COP and SOP $(\mathrm{p}=0.7)$.

\section{DISCUSSION}

This is the first nationwide study reporting the mean annual incidence of both COP and SOP. The period 1984-2003 was chosen because excellent computerised hospital records were available for both pathology and discharge diagnosis. As we performed a very thorough search, we believe that all relevant pathology reports were retrieved. We believe that this study includes all cases of OP diagnosed in Iceland during the study period.

Our results show that the mean annual incidence of COP and SOP is higher in Iceland than has previously been reported in a more limited population. ${ }^{7}$ This high incidence is established despite the fact that strict diagnostic criteria were used.

A study from Olmstead county in Minnesota found an age and sex adjusted annual incidence for OP of 0.85 per person years (95\% CI 0.24 to 1.45 per 100000$).^{7}$ A report on the incidence of interstitial lung disease (ILD) in Spain showed an estimated incidence of 7.6 per 100 000/year, and COP was estimated to account for $10.4 \%$ of the cases. ${ }^{8}$ A study from
Table 3 Mean annual increase in incidence of $\mathrm{OP}$ by age group

\begin{tabular}{ll}
\hline Age group (years) & $\begin{array}{l}\text { Mean }(95 \% \mathrm{CI}) \text { increase in } \\
\text { incidence }(\%)\end{array}$ \\
\hline$<60$ & $7.9(0.8$ to 15.5$)$ \\
$60-74$ & $8.2(2.0$ to 14.7$)$ \\
$75+$ & $8.1(7.6$ to 16.0$)$ \\
All & $8.1(4.1$ to 12.2$)$ \\
\hline
\end{tabular}

Table 4 Causes of death in patients with OP

\begin{tabular}{lccc}
\hline & Total & COP & SOP \\
\hline Organising pneumonia & 1 & 1 & 0 \\
Other lung diseases & 14 & 5 & 9 \\
Heart diseases & 11 & 5 & 6 \\
Cerebrovascular diseases & 4 & 0 & 4 \\
Haematological/oncological diseases & 13 & 6 & 7 \\
Other causes & 2 & 1 & 1 \\
Total & 45 & 18 & 27 \\
\hline
\end{tabular}

COP, cryptogenic organising pneumonia; SOP, secondary organising pneumonia.

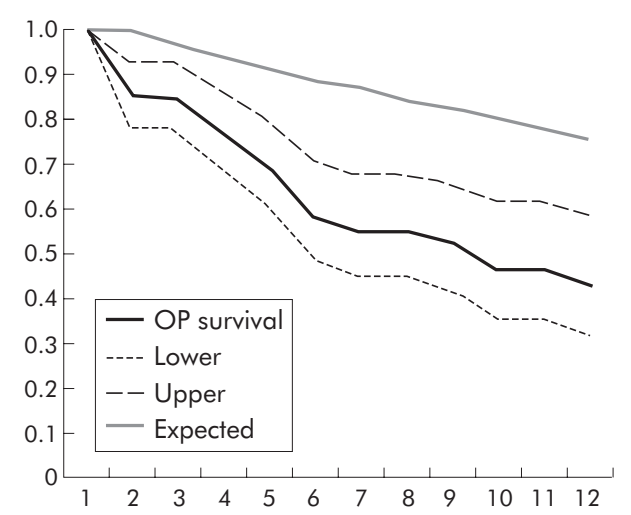

Figure 3 Kaplan-Meier curve of observed and expected survival in 104 patients with organising pneumonia (OP) in Iceland.

Germany showed that COP accounted for $6.8 \%$ of all cases of ILD $^{9}$ and in Italy it was responsible for $5 \%$ of all ILD cases. ${ }^{10}$ All of these studies are registries of patients seen by respiratory medicine centres. They are subject to selection bias and do not permit an estimate of the real incidence of COP. A study from Bernalillo county in New Mexico showed that the incidence of ILD was 32 per 100 000/year in men and 26 per 100 000/year in women. ${ }^{11}$ COP was reported in one $(0.5 \%)$ of 202 patients registered over 48 months. This is the only population based registry of all ILD published to date. It is, however, only a 4 year registry while ours covers a period of more than 20 years.

Overall, the incidence of OP increased during the study period. There could be a number of explanations for this. Firstly, physicians were not familiar with the disease during the first part of the study period. Secondly, the number of respiratory specialists in Iceland increased during the study period and more bronchoscopic examinations were performed. Thirdly, there was an increase in the use of medications and treatments that can cause SOP during the study period that could have led to more cases. However, there was a greater rise in the incidence of COP than of SOP during the period of the study. The fluctuations in the 
incidence of SOP may be explained, in part, by the fact that these patients are diagnosed and treated without a biopsy if physicians are confident of the diagnosis, which is less likely to occur if the diagnosis is unclear.

The age distribution in our study suggests that this is a disease that can occur from adolescence until old age, but most of the patients are elderly. These results are in agreement with the study by Cazzato et al from Italy. ${ }^{12}$ The median age in our study is similar to other studies. ${ }^{7}$

Our study shows that mortality is higher for patients with OP than in the general population, but it is similar for COP and SOP. There was a trend for a higher mortality in the SOP group than in the COP group, but the difference was not statistically significant. In a study performed at the Mayo Clinic, patients with SOP had a higher mortality rate than those with COP, but all cause mortality rates were similar. ${ }^{7}$ One possible explanation for the difference is that we did not classify the focal nodular variant as a separate entity. It is possible that having OP sensitises the patient for premature death. The systemic inflammation caused by OP could have an effect in different organs, including the cardiovascular system. $^{3}$

Our findings on the causes of death in patients with COP and SOP, with respiratory disease being the most common, are in agreement with those of Lohr et al. ${ }^{7}$ We found a low rate of mortality from the disease itself, but patients died from other respiratory causes and their underlying disease. It was common for patients in our study to have underlying lung disease when diagnosed with OP. After OP had been cured there was often continuing progression of the underlying disease (such as chronic obstructive pulmonary disease) that led to death. Deaths from treatment related complications were rare.

The strengths of our study include the fact that it was a nationwide study with no reference bias, covered a long period of registration, had excellent computerised records of all cases, and a long follow up period. Among the weaknesses are the small population of the country and the fact that it used causes of death on the death certificates which have been found to be inaccurate for interstitial lung diseases.. ${ }^{13}$ We used cases diagnosed with surgical biopsy that has been called "definite OP" and cases diagnosed with transbronchial biopsy obtained by flexible bronchoscopy. It has been suggested that these cases should be called "probable" cases. ${ }^{3}$ By doing this we get more cases and a more accurate estimate of the incidence of the disease than by using cases diagnosed with surgical biopsy alone. We agree with the suggestion that the diagnosis of OP can be made fairly accurately using transbronchial biopsy. ${ }^{14}$
In summary, the mean annual incidence of OP in this study was about 1.97 per 100 000, which is higher than some other studies have suggested. It is a disease that can occur in all age groups but is more common in the elderly. It leads to increased mortality compared with the general population, but there is no difference in mortality between the cryptogenic and secondary forms.

\section{ACKNOWLEDGEMENTS}

The authors thank all the pulmonary specialists in Iceland for sharing information for this study.

\section{Authors' affiliations}

G Gudmundsson, O Sveinsson, S Jonsson, H Frodadottir, Department of Respiratory Medicine, Allergy and Sleep, Landspitali University Hospital, Reykjavik, Iceland

H J Isaksson, Department of Pathology, Landspitali University Hospital, Reykjavik, Iceland

T Aspelund, The Icelandic Heart Association, Kopavogur, Iceland

This study was funded by Landspitali University Hospital Scientific Fund, Icelandic Thoracic Society Research Fund, and Oddur Olafsson Memorial Fund in Iceland.

Competing interests: none declared.

\section{REFERENCES}

1 Colby TV. Pathologic aspects of bronchiolitis obliterans organizing pneumonia. Chest 1992;102:38-43S.

2 American Thoracic Society/European Respiratory Society. Classification of the idiopathic interstitial pneumonias: international multidisciplinary consensus. Am J Respir Crit Care Med 2002;165:277-304.

3 Cordier JF. Cryptogenic organizing pneumonia. Clin Chest Med 2004;25:727-38.

4 Epler GR. Bronchiolitis obliterans organizing pneumonia. Arch Intern Med 2001;161:158-64.

5 Berry G. The analysis of mortality by the subject-years method. Biometrics 1983;39:173-84.

6 Therneau TM, Grambsch PM. Modeling survival data: extending the Cox model. New York: Springer, 2001.

7 Lohr RH, Boland BJ, Douglas WW, et al. Organizing pneumonia: features and prognosis of cryptogenic, secondary, and focal variants. Arch Intern Med 1997; 157:1323-9.

8 Xaubet A, Ancochea J, Morell F, et al. Report on the incidence of interstitial lung diseases in Spain. Sarcoidosis Vasc Diffuse Lung Dis 2004;21:64-70.

9 Schweisfurth H. Mitteilung der wissenschaftliches Arbeitsgemeinschaft fur die Therapie Lungenkrankheiten (WATL): Deutcher Fibrosereregister mit ernsten Ergebnissen. Pneumologie 1996;50:899-901.

10 Agostini C, Albera C, Bariffi F, et al. First report of the Italian Register for diffuse lung disorders. Monaldi Arch Chest Dis 2001;56:364-8.

11 Coultas DB, Zumwalt RE, Black WC, et al. The epidemiology of interstitial lung diseases. Am J Respir Crit Care Med 1994;150:967-72.

12 Cazzato S, Zompatori M, Baruzzi G, et al. Bronchiolitis obliterans-organizing pneumonia: an Italian experience. Respir Med 2000;94:702-8.

13 Coultas DB, Hughes MP. Accuracy of mortality data for interstitial lung diseases in New Mexico, USA. Thorax 1996;51:717-20.

14 Cordier JF. Organizing pneumonia. Thorax 2000;55:318-28. 